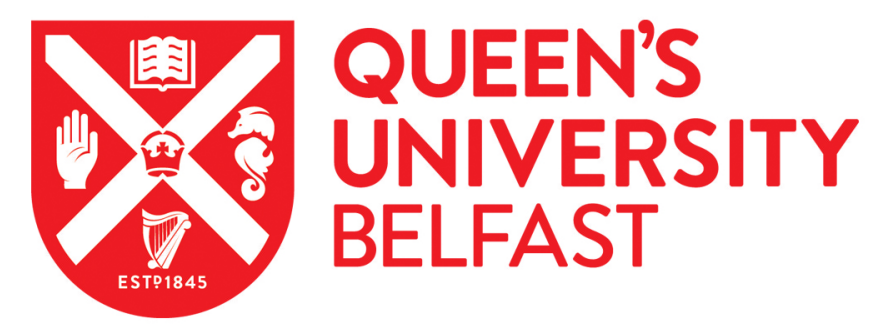

\title{
Multi-fidelity validation algorithm for next generation hybrid-electric vehicle system design
} Stevens, G., Early, J., Cunningham, G., Murtagh, M., Douglas, R., \& Best, R. (2019). Multi-fidelity validation
algorithm for next generation hybrid-electric vehicle system design. Proceedings of the Institution of Mechanical
Engineers, Part D: Journal of Automobile Engineering, 233(13), 3438.

https://doi.org/10.1177/0954407018825015

Published in:

Proceedings of the Institution of Mechanical Engineers, Part D: Journal of Automobile Engineering

Document Version:

Peer reviewed version

Queen's University Belfast - Research Portal:

Link to publication record in Queen's University Belfast Research Portal

Publisher rights

Copyright 2018 IMechE 2019.

This work is made available online in accordance with the publisher's policies. Please refer to any applicable terms of use of the publisher.

\section{General rights}

Copyright for the publications made accessible via the Queen's University Belfast Research Portal is retained by the author(s) and / or other copyright owners and it is a condition of accessing these publications that users recognise and abide by the legal requirements associated with these rights.

Take down policy

The Research Portal is Queen's institutional repository that provides access to Queen's research output. Every effort has been made to ensure that content in the Research Portal does not infringe any person's rights, or applicable UK laws. If you discover content in the Research Portal that you believe breaches copyright or violates any law, please contact openaccess@qub.ac.uk. 


\title{
Multi-fidelity Validation Algorithm for Next Generation Hybrid Electric Vehicle System Design
}

\author{
Gary Stevens ${ }^{\mathrm{a},}$, Juliana Early ${ }^{\mathrm{a}}$, Geoff Cunninghama, Martin Murtagha, Roy \\ Douglas $^{\mathrm{a}}$, Robert Best ${ }^{\mathrm{b}}$ \\ ${ }^{a}$ School of Mechanical and Aerospace Engineering, The Queen's University of Belfast, \\ Belfast, BT9 5AG, Northern Ireland \\ ${ }^{b}$ Wrights Group Ltd. Galgorm Industrial Estate, Fenaghy Rd, Galgorm, Ballymena BT42 \\ $1 P Y$.
}

*Corresponding author Tel: +442890974944 E-mail address: gstevens01@qub.ac.uk

\begin{abstract}
With the evolution of increasingly complex hybrid electric vehicle powertrains the process of creating validated system models has become progressively more difficult to achieve. Increasing levels of confidence in how instantaneous vehicle energy states are captured is needed to take full advantage of the fuel consumption and emissions reduction potential of the vehicle to move towards more sustainable transportation systems. While many strategies for model validation exist, the majority rely on ascertaining comparisons with global system characteristics, for instance total fuel consumption over a fixed driving event. However, these methods do not
\end{abstract}


necessarily account for the rapidly fluctuating energy states which need to be understood to optimise the vehicle's energy management strategy. The current work proposes a new validation approach which captures these instantaneous characteristics taking advantage of the high signal sampling rates available from modern data acquisition equipment rather than relying on drive cycle average or cumulative global behaviours. The method proposed provides a holistic view of the behaviours demonstrated by the vehicle model and identifies regions of poor system validation targeting areas for further model refinement. The algorithm is demonstrated on a new post-transmission, parallel mild-hybrid electric bus. The model was developed in the MATLAB Simulink modelling environment. The validation algorithm is tested against vehicle dynamometer and test track data. With an increasing volume of mild and full hybrid vehicle configurations emerging, validation strategies such as the one proposed here are increasingly important for the design of energy management strategies to deliver the full potential benefits of the vehicle. The algorithm is proposed in a step by step method which can be automated to limit required user input.

Keywords: Simulation, Validation, Powertrain, Modelling, Hybrid, Bus 


\section{Introduction}

It is commonplace in nearly all vehicle engineering applications to develop a mathematical model, or suite of models, which can represent the various behaviours demonstrated by a given vehicle configuration across a series of driving events. Regulators, manufacturers and buyers often wish to compare alternative powertrain technologies without having to measure performance directly [1]. This can be achieved through the use of simulation models. In the context of powertrain development, the use of models of this type can significantly reduce the time and/or cost of associated product development and prototyping [2]. Accurate fuel consumption estimates can also encourage increased investments in energy efficient technologies by both manufacturers and consumers [3]. In the passenger bus sector, the area of the current study, the complexity of the vehicle development process has increased significantly over the last decade. Rapidly evolving customer demands for ever more fuel-efficient configurations is matched in pace with increasingly stringent legislative requirements, such as Euro VI emission legislation for heavy duty vehicles [4] introduced in 2014 [5]. These industry drivers are present as a result of increased global desire to move towards more sustainable transportation systems. Key to the success of these development processes is a well-developed and validated simulation model. However, there is still no commonly accepted verification and/or validation process adopted within the vehicle modelling domain, and practices reported in literature can range from simplistic global schemes to extremely refined levels of fidelity 
[6]-[10]. Furthermore, while various standards have been developed in some domains (for instance [11], [12]), there is no harmonised framework for validation of a vehicle model when a strong understanding of the underlying system dynamics is needed. This need for detailed understanding of the system performance has become increasingly prevalent in the vehicle engineering domain over recent years with the advent of hybrid and fully electric vehicle configurations, particularly in cases where the simulation results are directing the development of energy management strategies. For complex hybrid architectures, such as parallel hybrids, a detailed energy management strategy is required to control the blend of power between the electrical systems and the internal combustion engine. For the hybrid-electric vehicles studied here, this detailed understanding of the link between the underlying system dynamics and the optimisation of the energy management strategy is necessary to maximise the fuel saving potential of the vehicle whilst maintaining a high level of confidence in the overall energy storage system performance.

Specific to vehicle powertrain modelling, verification and validation processes are typically iterative in nature. Verification processes determine if the model is functioning correctly with respect to laws of physics i.e. conservation of energy, momentum, mass etc. Typically, this model verification process should also ensure that implemented control systems are functioning correctly with respect to their underlying mathematical logic. The subsequent validation procedure will typically take this one step further to ensure that the 
model is suitably representative of the 'real-life' system. Central to this process is the notion of the 'data validity', as discussed by Sargent [13]. In most vehicle engineering applications, validation data sets are elicited through experimentation or testing of real components but can be heavily dependent on the user having a thourough understanding of the type, nature and rate of data acquisition required to achieve a high-quality validation of their model.

The typical objectives of vehicle model validation studies can range from evaluating the suitability of the model calculating fuel consumption characteristics through to an evaluation of performance over a specific driving event, ultimately providing an assessment of the credibility of the simulation for a specified use [14]. These characteristics tend to either be 'global', for example, total fuel consumption or comparison of average velocity/acceleration rates over a specified drive cycle, or 'local', when a specific vehicle behaviour is of interest, such as instantaneous power demand during an acceleration event. An extensive range of local and global model validity measures have been developed through numerous studies. For example, qualitative 'Face Validity' is achieved by an expert user who can 'validate' the model based on their knowledge of how the real system operates under given conditions [15]-[21]. More advanced quantitative statistical techniques can range from evaluation of percentage error and root mean square errors between simulation and experimental results for given characteristics [22]-[26], through to more comprehensive evaluation of signal correlation 
such as use of the 'R-Squared' method as shown by Wang and Rakha [27] and some methods which use the frequency domain in their analyses to capture high frequency transient phenomena [28], [29]. The single metric approaches have also been extended to multi-step validation procedures, for example in the approach of Klemmer et al. [30] where a two stage method for validation of a vehicle model based on a signal correlation (SC), a correlation rating (CR) and a validation coverage rating (VC) was presented. However, while there have been a range of methods which can quantify measures of the model validity, most are still highly subjective and performed 'one signal at a time'. While removing subjectivity entirely from any validation process would be extremely difficult to achieve, it would be desirable to remove subjective input from the user as far as possible. Furthermore, despite the range of methods and techniques which have been proposed, there is still a lack of work in the public domain relating to validation of full powertrain simulation models. This may, in part, be due to the expense in acquiring suitable experimental validation data for these activities, and a result of the complexity in interpreting the vast array of signals within the system. The current work is proposing a new a multi-fidelity validation process, demonstrated on a mild-hybrid passenger bus powertrain model. The algorithm is capable of capturing both detailed local and global measures into a convenient set of easily interpretable validation characteristics. Section 2 will discuss the vehicle model used to illustrate the validation procedure as well as outlining the general form of the procedure. The proceeding subsections will discuss each 
step in the procedure with reference to real world test data sets and provide examples of application to the mild-hybrid passenger bus model. Section 3 will discuss the validation procedure in detail with results from application to the current work. Section 4 will critique the proposed validation procedure and finally section 5 will summarise the conclusions from the current work.

\section{Model and Validation Procedure Outline}

A forward-facing model of the mild hybrid bus configuration developed by Stevens et al. [31] has been used in this work. A post-transmission, parallel mild-hybrid electric passenger bus configuration has been developed, verified and further validated using the methodology proposed in this paper. Figure 1 shows a schematic diagram of the proposed mild-hybrid vehicle configuration in comparison to a conventional powertrain architecture. It should be noted that the hybrid vehicle architecture is capable of operating as a fully electric vehicle for short periods and could therefore also be classified as a full hybrid. The vehicle modelled is a double deck passenger bus with gross vehicle weight of 18 tonnes, intended for urban inner-city usage. As the highest sampling rate available from the vehicle Controller Area Network (CAN) during vehicle testing is $100 \mathrm{~Hz}$ this was also selected as the default sampling rate for the vehicle simulation. Any data that was not logged at $100 \mathrm{~Hz}$ during the vehicle testing phase (largely limited to signals with low rates of change and therefore higher frequency sampling would be inappropriate) was upsampled during pre-processing to ensure compatibility with simulation sampling rates. 
The sampling rate selected for use in the validation process is at the discretion of the user. Suitable judgement should be used to determine the correct sampling rate based on the nature of the signals being investigated, for example higher rates should be used for an assessment of power electronics behaviour in comparison to component thermal characteristics which would exhibit behaviour on a much larger timescale. Across the vehicle powertrain at any point in time, approximately 600 discrete signals are being logged at various system and sub-system levels across the architecture, providing a level of insight into the system complexity and the potential issues with performing detailed validation. With the number of different signals to manage, many of which are highly time dependant, it can become challenging to identify signals with poor correlation and more importantly the likely cause.

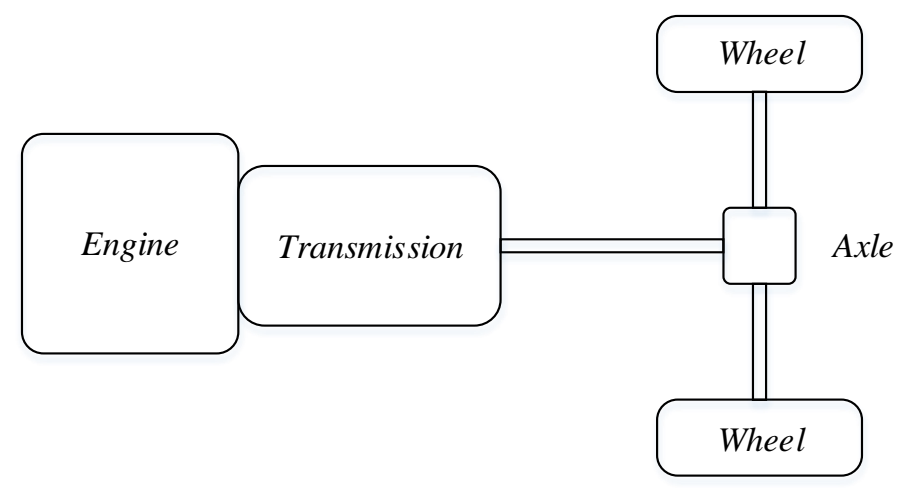

(a) 


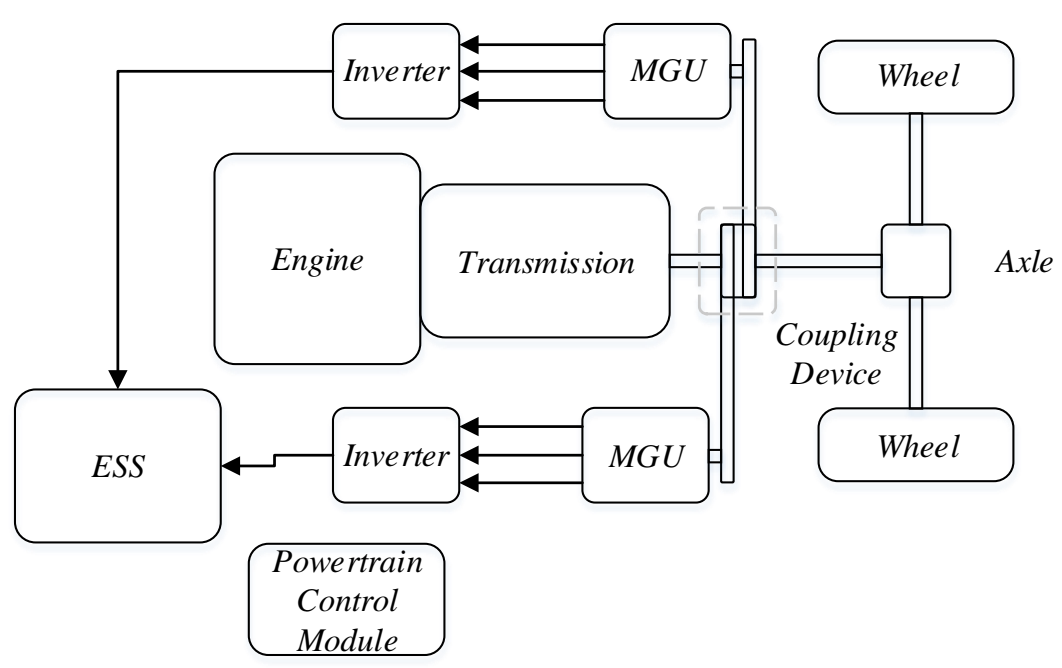

(b)

Figure 1 - Comparison of bus vehicle configuration from (a) conventional to (b) mildhybrid

To overcome this inherent complexity, the proposed validation process has been developed around a multi-fidelity algorithm which yields a vector of metrics to represent the validation state of the vehicle. In this case the validation is being performed with the mild-hybrid vehicle model simulating results on an MLTB drive cycle as significant experimental test data was available to prove the proposed method. The MLTB cycle is a bespoke driving cycle created by Millbrook Proving Ground in England specifically for the passenger bus industry. It is representative of a bus route which travels from the outskirts of London into the city centre with a section of higher velocity acceleration and deceleration events at the start of the drive cycle, transitioning into a similar pattern of lower velocity driving events. It is typically regarded as a challenging driving route to achieve good vehicle performance across. Figure 2 shows the velocity profile of the 
MLTB drive cycle.

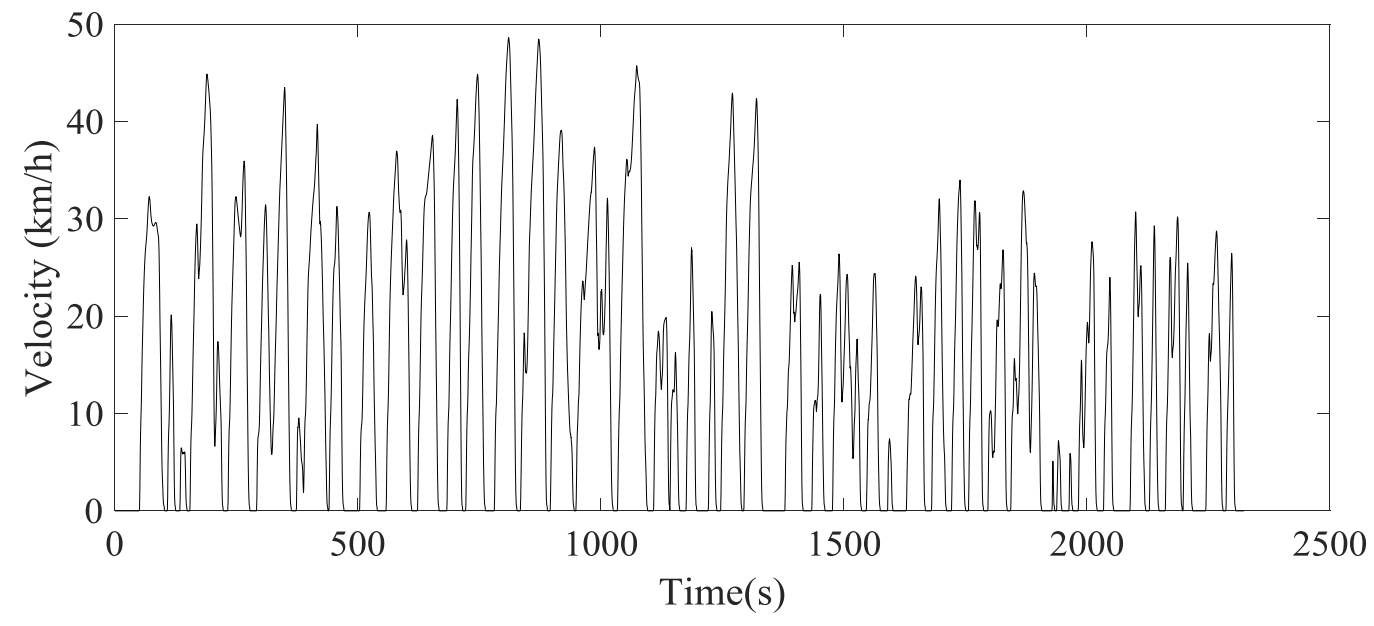

Figure 2 - MLTB drive cycle

Note that from the outset it is assumed that the model has already been verified; in that the results abide by physical laws of conservation of momentum and energy and that underlying model kinematics have been correctly implemented. The following validation procedure is implemented for a mild-hybrid vehicle architecture but it should be noted that the proposed algorithm could equally be applied to any complex dynamic system. To perform the validation, a series of formalised steps have been identified, which are described in detail in Sections 3.1 to 3.7 :

1. Identification of the objective signals for the model.

2. Signal decomposition to create validation signal set(s).

3. Evaluation of signal behavioural characteristics.

4. Aggregation of results to produce a global validation metric. 


\section{Detailed validation procedure}

For clarity in this paper, a smaller subset of local and global model signals is discussed that are suitable for validating the model's fuel consumption prediction characteristics, but it should be noted that the validation procedure can be applied across all aspects of the vehicle model depending on the characteristics of interest. For each signal evaluated a comprehensive set of experimental data sets was available for validation identified as the 'reference' data set or signal.

\subsection{Selection of validation signals}

A signal (or signal(s)) within the vehicle is identified as the 'objective signal(s)'. The choice of the objective signal(s) is dependent on the modelling and simulation objectives i.e. the purpose for which the model was created. This objective signal is decomposed to produce a set of validation signals, $\Gamma_{\mathrm{i}}$, where $i=\{1,2,3 \ldots n\}$, i represents each signal set and $n$ is the total number of sets identified within the model. For the hybrid-electric vehicle, these objective signals are typically global characteristics of the vehicle, such as cumulative fuel consumption. Each objective signal is subsequently decomposed through a signal identification process to establish several validation signals for the model, $\gamma_{i, j, k}$, where $i$ denotes membership to the parent $\Gamma_{i}$ signal set, $j$ denotes the depth or level of decomposition of the signal relative to the objective parent signal, and $k$ denotes the position of the validation signal within its own decomposition level. An individual signal may belong to more than one validation signal set, and that the objective signal is also the 
first signal in the validation set, $\gamma_{i, 1,1}$. Each of these validation signals is typically noncomplex and continuously varying with respect to time, displaying appreciable changes in magnitude across the time range of interest. A generic validation signal set is shown in Figure 3 to illustrate the notation system.

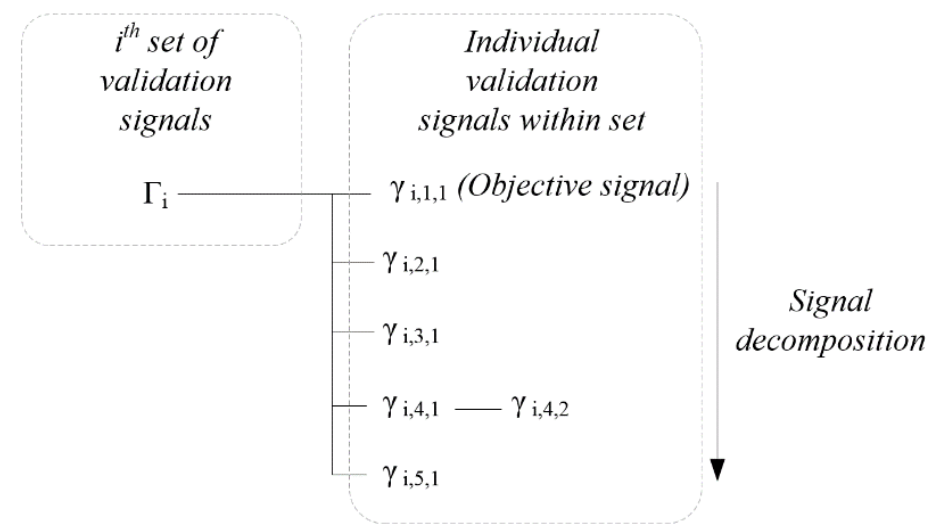

Figure 3 - Objective signal decomposition into validation signal set

As a simple example of this process, cumulative fuel consumption $\left(F C_{c u m}\right)$, is selected as the objective signal, $\gamma_{1,1,1}$ and assigned to the first signal set, $\Gamma_{1}$, as shown in Figure 4. Cumulative fuel consumption is calculated through the integration of the instantaneous fuel consumption $\left(F C_{\text {inst }}\right)$, over the driving event of interest spanning a time range $t=t_{0}$ to $t_{\text {end }}($ Equation 1).

$$
\gamma_{1,1,1}=F C_{\text {cum }}=\int_{t_{0}}^{t_{\text {end }}} F C_{\text {inst }} d t
$$

Continuing the decomposition process, instantaneous fuel consumption is a function of instantaneous engine speed $\left(N_{\text {eng }}\right)$ and engine torque $\left(T_{\text {eng }}\right)$ as shown in Equation 2. 


$$
\gamma_{1,2,1}=F C_{\text {inst }}=f\left(N_{\text {eng }}, T_{\text {eng }}\right)
$$

Engine speed is therefore added to the set as $\gamma_{1,3,1}$ and engine load torque is added to the same set as $\gamma_{1,3,2}$. This process is continued for each objective signal in turn until no further depth of decomposition can be achieved. A second set was included which contained only the vehicle velocity, $v$, as this was also deemed important to the assessment of model validity as including this signal in the process ensures that the simulation and real-world vehicle are performing the same driving events. The new signal set is illustrated in Figure 4.

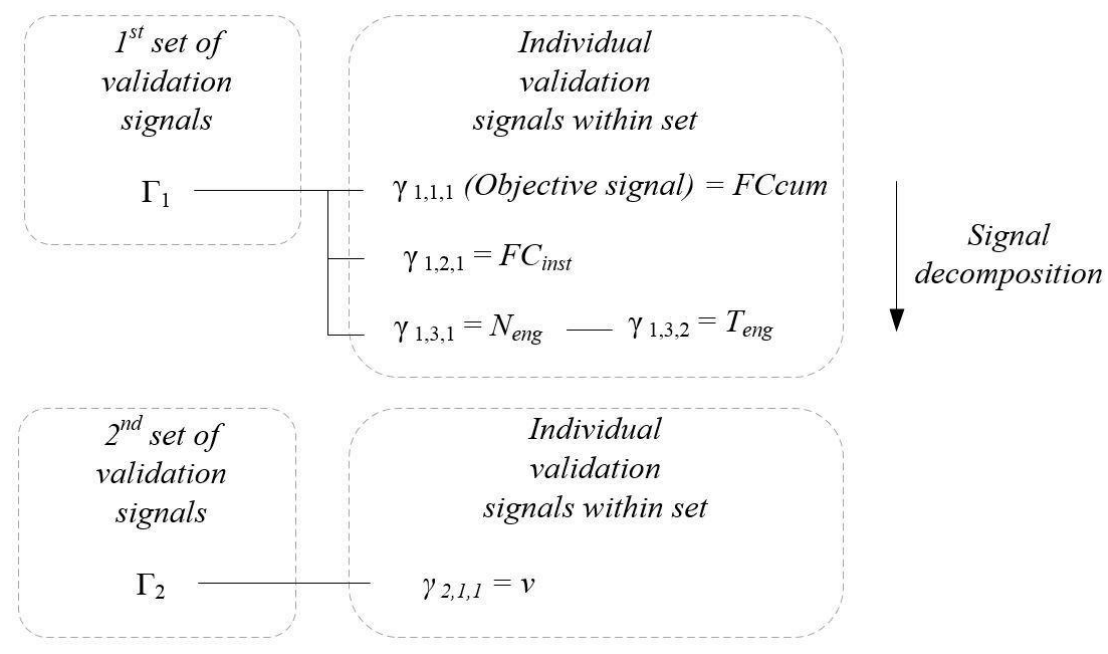

Figure 4 - Validation Signal Sets

Each of the validation signals is evaluated against a set of behavioural characteristics, $\Lambda$, where each discrete characteristic is denoted as $\lambda_{i, j, k, q}$. Here, $i, j$, and $k$ relate to the position of each signal within the decomposition sequence and $q=\{1,2,3 \ldots m\}$ is an 
identifier for the metric that is used to quantify each behavioural characteristic. A set of seven behavioural characteristics $(m=7)$ are proposed here, identified as capturing all the necessary facets of the system behaviours in order to provide sufficient confidence in the model validation. These behavioural characteristics are shown in Table 1.

Table 1 - Validation Behaviour Set

\begin{tabular}{cc}
\hline Behaviour & Description \\
\hline$\lambda_{i, j, k, 1}$ & Time Domain Lead/Lag Behaviour \\
$\lambda_{i, j, k, 2}$ & First Order Derivative \\
$\lambda_{i, j, k, 3}$ & Mean Absolute Error (MAE) \\
$\lambda_{i, j, k, 4}$ & Root Mean Square Error (RMS) \\
$\lambda_{i, j, k, 5}$ & $\Delta E=\left|E_{r m s}-E_{\text {mae }}\right|$ \\
$\lambda_{i, j, k, 6}$ & Standard Deviation of Error \\
$\lambda_{i, j, k, 7}$ & Distribution \\
\hline
\end{tabular}

It is assumed that prior to any mathematical evaluation, a visual inspection of the qualitative characteristics of the validation sets would have been undertaken. While each of the behaviours are discussed individually, the calculation process is automated to ensure that there is limited user interaction required. 


\section{2. $\lambda_{i, j, k, 1}$ Time domain leads/lags and Signal Correlation}

The first behavioural characteristic is the time domain lead/lag through use of a correlation analysis. The signal correlation can be assessed using a cross correlation function which shifts a signal of interest by a given amount in the time domain $(\tau)$ and compares the extent of overlap to a fixed reference signal (in this instance 'reference' will always refer to real vehicle test data). The value of $\tau$ is then adjusted iteratively and comparisons made between the simulation and reference signals for each given value of $\tau$. The strongest correlation occurs at the point where the overlap between the two signals is maximised, referred to as a correlation peak. By identifying the necessary time shift that needs to be applied to the signal of interest to achieve maximum signal correlation, lead and lag phasing errors can be identified and necessary model adjustments made to remove the error, if desired. Cross correlation for continuous functions is described by Equation 3 .

$$
(f * g)(\tau) \stackrel{\operatorname{def}}{=} \int_{-\infty}^{\infty} f^{*}(t) g(t+\tau) d t
$$

where $f$ and $g$ are arbitrary functions to be cross correlated, $f^{*}(t)$ denotes the complex conjugate of $f(t)$ (for functions with no imaginary components this is equal to the function itself), and $\tau$ is the aforementioned time shift value. The results from the cross-correlation function have been normalised so that the autocorrelation of the two signals (the cross correlation of a signal with itself) at zero time lead or lag results in a value of one. Therefore, a cross-correlation result is better if the correlation is closer to one and the 
corresponding time lead or lag is close to zero. As an example of this, the vehicle velocity cross-cross correlation plot is shown in figure 5.

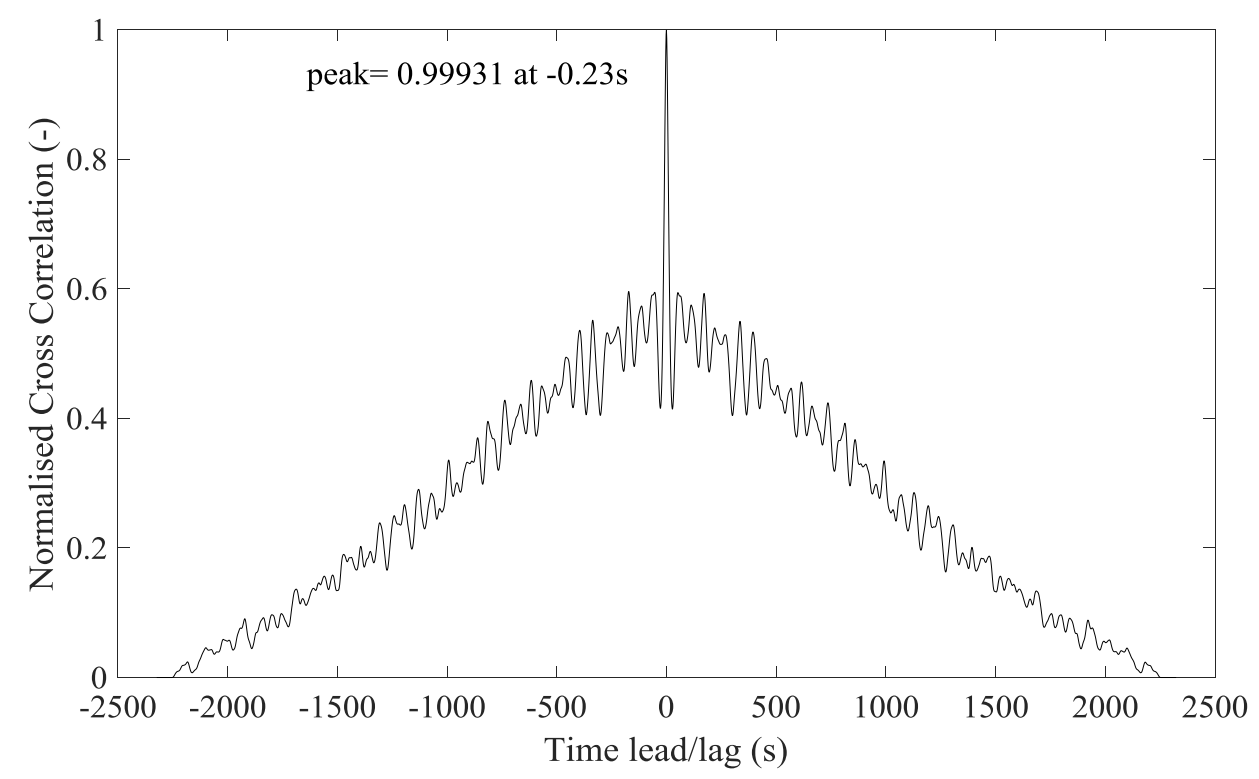

Figure 5 - Vehicle velocity cross correlation plot

The velocity cross correlation plot shows that the peak of the function is at 0.99931 at 0.23 seconds (note that the minus indicates a time lag i.e. the simulated signal lags the reference signal). This is interpreted as a $99.931 \%$ correlation rating at a time lag of 0.23 seconds. The correlation at 0 seconds time lag for the same velocity signal was found to be 0.99903 or $99.903 \%$. The cross-correlation rating of 0.99903 for vehicle velocity now gives a value for the first behavioural characteristic metric for the first signal in the second set, $\lambda_{2,1,1,1}=0.99903$. As there is a small percentage difference between the correlation 
rating at $-0.23 \mathrm{~s}$ and $0 \mathrm{~s}$ time lag, this illustrates that there is a negligible effect from the phasing delay between the simulation results and real vehicle test results; this helps to identify those errors arising from time-delays rather than any mathematical anomalies as shown in Figure 6.

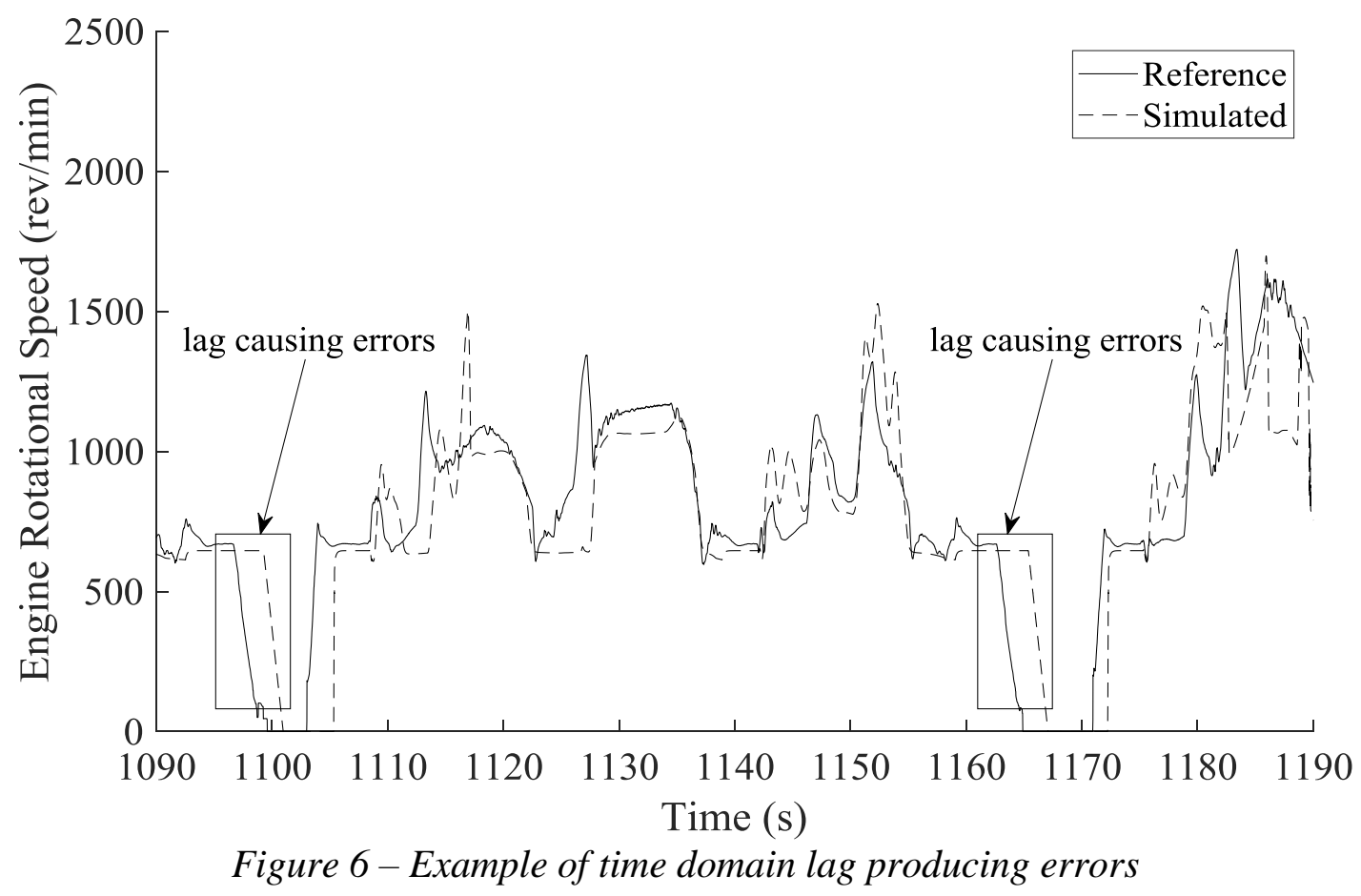

\section{3. $\lambda_{i, j, k, 2}$ First Order Derivatives $\left(d \gamma_{i, j, k} / d t\right)$}

The numerical first order derivative of each of signal (simulated and reference) is then calculated to determine their respective rates of change at each instance in time and to ascertain matching in directionality for signal movement (positive/negative rates of change). For a signal comprising of $n$ samples, the first order derivative is calculated for 
each sample $(i)$ in the closed interval $i=[2$, end-1] using the preceding sample $(i-1)$ and the proceeding sample $(i+1)$, where 'end' is the last sample point in the vector (Equation 4).

$$
\left.\frac{d y}{d t}\right|_{i=2} ^{i=\text { end }-1} \cong \frac{y_{i-1}-y_{i+1}}{t_{i-1}-t_{i+1}}
$$

where $y$ is the magnitude of the given signal and $t$ is the time. The first and last sample points are set to zero for this method, but providing the time step used in the simulations are small (in the current example, a time step of 0.01 seconds is used) the error introduced is negligible. Once the first order derivatives are calculated, the time dependent directionality of each of derivative for the simulation and the reference data is directly compared and a logical vector of true and false values returned (true when the directionalities match, and false otherwise). The fraction of the simulation sample steps where there is directionality matching can then be found providing a value for $\lambda_{i, j, k, 2}$. Figure 7 shows a section of the $1^{\text {st }}$ derivative plot for the instantaneous fuel consumption signal for the hybrid vehicle configuration, highlighting signal directionality match and mismatch. 


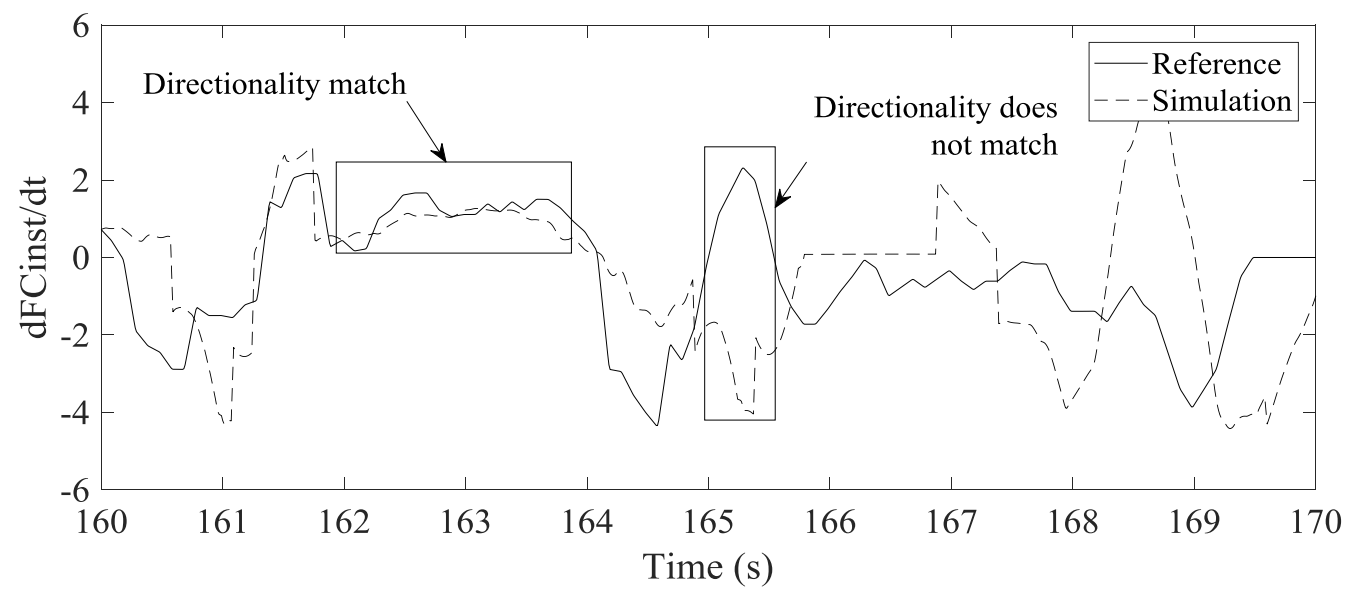

Figure 7 - Sample of instantaneous fuel consumption signal showing directionality match and mismatch

For the current driving cycle, for the instantaneous fuel consumption signal the directionality match fraction was 0.6818 . If the phasing error from the first step was corrected for, this matching increased to 0.70161 ; by considering these metrics in this fashion it ensures that subtleties in the behaviour such as this are revealed. While it is difficult to achieve a high level of directionality match on signals with high sampling frequencies, it is undesirable to smooth the data as some of the important time dependent characteristics in the results would be lost. The value of 0.6818 is now assigned to the second behavioural characteristic for the second signal in the first set, $\lambda_{1,2,1,2}$.

\section{4. $\lambda_{i, j, k, 3}, \lambda_{i, j, k, 4}$ and $\lambda_{i, j, k, 5}$ Mean Absolute Error, Root Mean Square and $\Delta E$}

At this stage the user can now assess the magnitude of the errors between the simulated and reference signals using a metric derived from the mean absolute error (MAE) and root mean square error (RMS). In the majority or reported validation processes, only the MAE and RMS characteristics are evaluated. However, it is proposed here that these two 
measures may not accurately reflect the system dynamics. Here, the absolute difference between the RMS and MAE errors is used to establish the severity of any anomalies that exist between the reference and the simulated data, which is typically what the user hopes to achieve from this type of error analysis. Equations for mean absolute error, root mean square error and the difference between these two error metrics, referred to as $\Delta E$, are shown in equations 5, 6 and 7 respectively.

$$
\begin{gathered}
E_{M A E}=\frac{1}{N} \sum_{i=1}^{N}\left|y_{\text {ref }, i}-y_{\text {sim }, i}\right| \\
E_{R M S}=\sqrt{\frac{1}{N} \sum_{i=1}^{N}\left(y_{\text {ref }, i}-y_{\text {sim }, i}\right)^{2}} \\
\Delta E=\left|E_{\text {rms }}-E_{\text {mae }}\right|
\end{gathered}
$$

where $y_{r e f, i}$ is the value of the reference signal at the $i^{\text {th }}$ time step, $y_{s i m, i}$ is the value of the simulated signal at the $i^{\text {th }}$ time step and $N$ is the total number of time steps. By evaluating $\Delta E$, the user can establish not only how well the two data sets match in terms of time domain error average values but also extract a metric for describing the presence (or absence) of anomalies within the results. Figure 8 shows a generic example of reference data and two different sets of simulation results. 


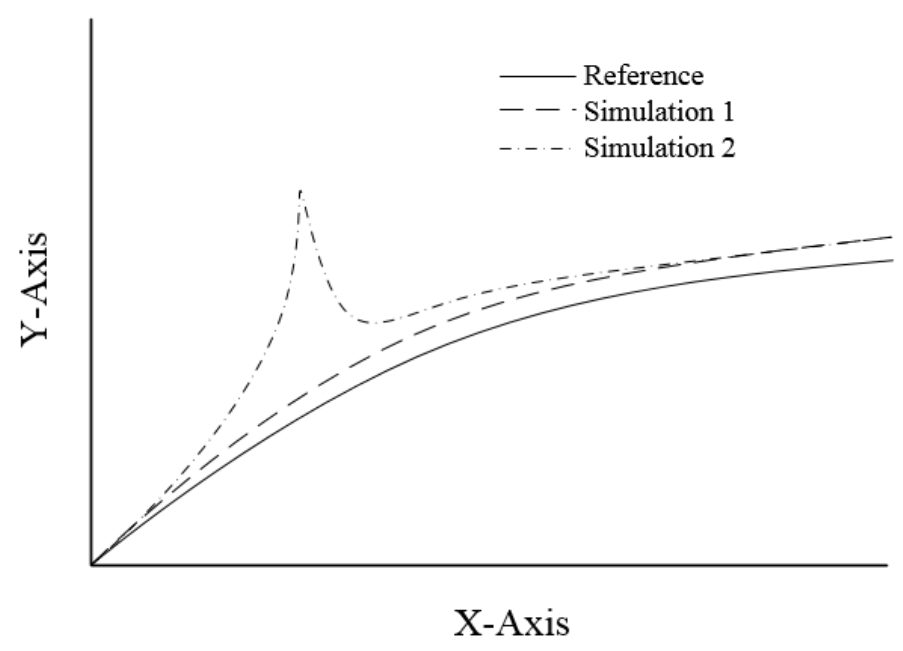

Figure 8 - Example of anomalous and non-anomalous signals

For Simulation 1, $E_{R M S} \cong E_{M A E}$ and therefore, $\Delta E$ should be relatively close to zero. The user can infer from this that there are scarce extreme anomalies within the results even though errors are still present. On the other hand, if the same calculations are performed for Simulation 2 it will be found that $E_{R M S} \gg E_{M A E}$ and therefore $\Delta E$ will be significant. This is caused by the anomalous 'spike' that occurs in the results for Simulation 2. The user can then infer that significant anomalies exist, even if overall trends in data are similar. When applied to the vehicle simulation, the results in Table 2 below were obtained (only velocity and instantaneous fuel consumption are presented for discussion). 
Table 2 - Mean, Root Mean Square and $\Delta$ E Error Calculations

\begin{tabular}{ccc}
\hline Metric & Velocity $(\mathbf{k m} / \mathbf{h})$ & $\boldsymbol{F C}_{\text {inst }}(\mathbf{g} / \mathbf{s})$ \\
\hline$E_{M A E}\left(\lambda_{i, j, k, 3}\right)$ & 0.6035 & 0.56119 \\
$E_{R M S}\left(\lambda_{i, j, k, 4}\right)$ & 1.0679 & 1.08 \\
$\Delta E\left(\lambda_{i, j, k, 5}\right)$ & 0.46442 & 0.51883 \\
\hline
\end{tabular}

For the velocity signal, the $\Delta E$ is small relative to the scale of the signal $(0.5 \%$ of the full range of the velocity signal). As the value of $\Delta E$ is small (relative to the scale of the signal) the extent of anomalies in the signal is low. Considering the instantaneous fuel consumption signal, the value of $\Delta E$ of $0.51883 \mathrm{~g} / \mathrm{s}$ would also appear to be quite small. However, this represents $4.5 \%$ of the full range of the signal, almost a full order of magnitude larger than observed in the velocity signal. Therefore, relative to the size of the signal, there are more anomalies present within the simulation of the instantaneous fuel consumption for the vehicle model than there are in the vehicle velocity signal. These results are further normalised. MAE, RMS and $\Delta E$ error normalisations were conducted as follows in equations 8, 9 and 10 and subsequently assigned to $\lambda_{i, j, k, 3}, \lambda_{i, j, k, 4}$ and $\lambda_{i, j, k, 5}$.

$$
\lambda_{\mathrm{i}, \mathrm{j}, \mathrm{k}, 3}=1-\left(E_{M A E} \times \frac{1}{y_{\text {ref }, \text { max }}-y_{\text {ref }, \text { min }}}\right)
$$




$$
\begin{gathered}
\lambda_{\mathrm{i}, \mathrm{j}, \mathrm{k}, 4}=1-\left(E_{R M S} \times \frac{1}{y_{\text {ref }, \text { max }}-y_{\text {ref }, \text { min }}}\right) \\
\lambda_{\mathrm{i}, \mathrm{j}, \mathrm{k}, 5}=1-\left(\Delta E \times \frac{1}{y_{\text {ref }, \text { max }}-y_{\text {ref }, \text { min }}}\right)
\end{gathered}
$$

where $y_{\text {ref,max }}$ and $y_{\text {ref,min }}$ are the maximum and minimum values of the reference signal corresponding the simulated signal for which the error metric was calculated.

\section{5. $\lambda_{i, j, k, 6}$ Standard deviation, Error distribution and Cumulative distribution function}

For each signal in turn, the standard deviation and cumulative distribution of errors in the simulation are calculated. The error was calculated as $y_{r e f}-y_{s i m}$. This enables the error distribution to contain negative and positive values. Again, for the purpose of demonstration, the instantaneous fuel consumption signal is considered. The histogram has an approximate Gaussian, or normal, distribution around the mean as shown in Figure

9. The ideal shape for these two plots would be for the histogram to have all its values around zero and the cumulative distribution function to have a normalised cumulative count of zero until an error value of zero is reached, and then instantaneously step to a normalised cumulative count value of one. 

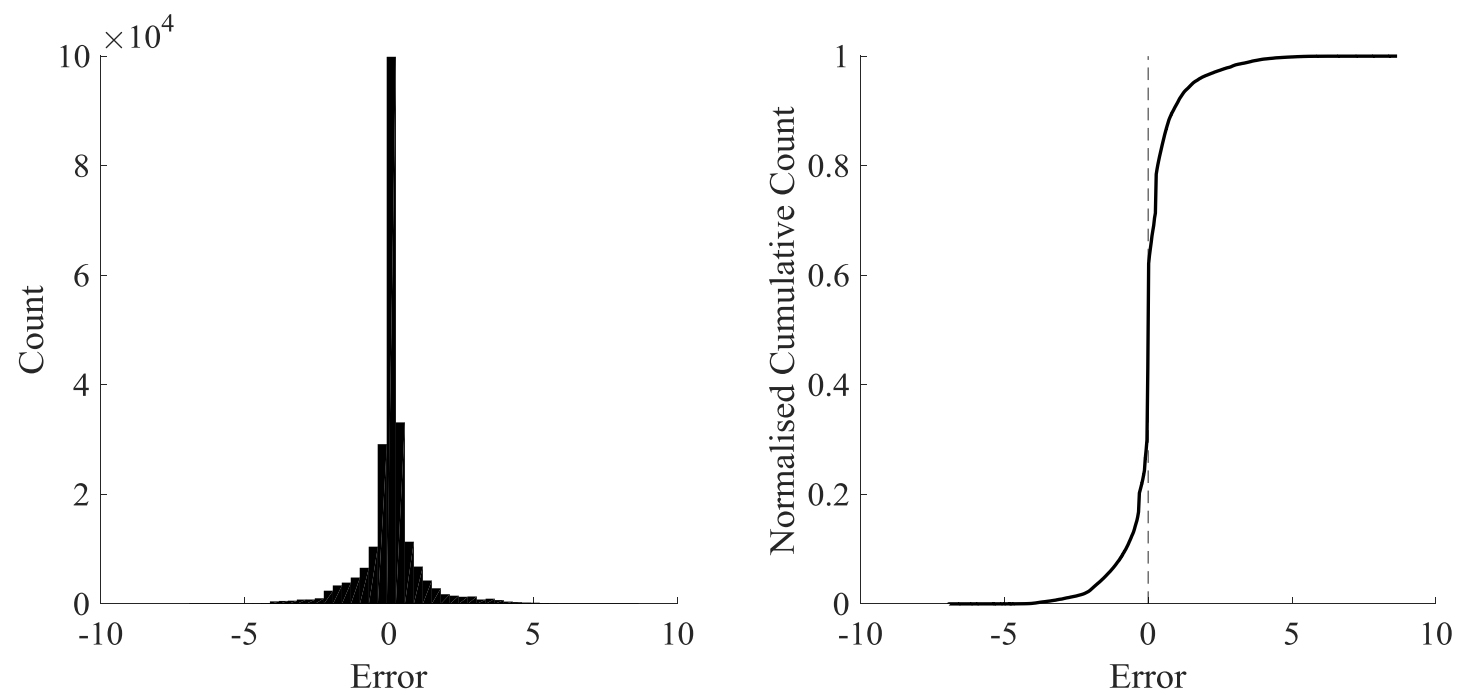

Figure 9 - Instantaneous fuel consumption error histogram and cumulative distribution function

For the instantaneous fuel consumption, the standard deviation $(\sigma)$ is $1.0781 \mathrm{~g} / \mathrm{s}$, where

$$
\sigma=\sqrt{\frac{1}{N-1} \sum_{i=1}^{N}\left|A_{i}-\mu\right|^{2}}
$$

$A$ is the variable vector made up of $N$ sample points and $\mu$ is the sample mean. Again, a normalisation must be performed to make the standard deviation results suitable for comparison. Here, the normalisation is calculated as shown in Equation 12.

$$
\lambda_{i, j, k, 6}=1-\left(\sigma \times \frac{1}{y_{\text {ref, } \text { max }}-y_{\text {ref }, \text { min }}}\right)
$$




\section{6. $\lambda_{i, j, k, 7}$ Inter-layer emergent behaviour identification}

The penultimate step is to identify any emergent behaviour that may (or may not) arise as a result of any combinatorial processing of signals and propagation through the model to higher levels. This is achieved by looking at the errors of lower level signals and comparing to the signal at the level directly above in the decomposition framework. This allows assessment of how the error has propagated, i.e. became larger because of mathematical combination or smaller due to 'averaging out' effects. The lowest signals within each set, i.e. the signals at the lowest level of decomposition, will not have a result for this behavioural characteristic associated with them because there is no layer below these for which to assess any emergent behaviour. This inter-layer behaviour is quantified by assessing the average of the behavioural characteristics for a signal at level $\mathrm{j}+1$, performing the same average for a signal at level $\mathrm{j}$ and then assessing the difference between the two. This process is generalised in Equation 13.

$$
\lambda_{\mathrm{i}, \mathrm{j}, \mathrm{k}, 7}=1-\left[\frac{1}{Q \times n} \sum_{q=1}^{Q} \lambda_{\mathrm{i}, \mathrm{j}, \mathrm{k}_{\mathrm{n}}, \mathrm{q}}-\frac{1}{Q \times n} \sum_{\mathrm{q}=1}^{Q} \lambda_{\mathrm{i}, \mathrm{j}+1, \mathrm{k}_{\mathrm{n}}, \mathrm{q}}\right]
$$

where $\mathrm{Q}$ is the total number of behavioural characteristics preceding the inter-layer emergent behaviour characteristic. $k_{n}$ is the subset of signals of $k$, where $n$ denotes the number of signals in this subset. $k_{n}$ is used due to the possibility that at any level of signal decomposition, $\mathrm{j}$, multiple signals could exist and therefore the average should be computed for all the characteristics for all the signals at that level. To show an example of 
this the emergent behaviour will be analysed between layers 2 and 3 of the cumulative fuel consumption signal set i.e. between engine torque and speed at level 3 and instantaneous fuel consumption at level 2. Table 3 shows the results from the calculation from each of the previously evaluated behavioural characteristics from these signals and the results from equation 13 assigned to the last behavioural characteristic, $\lambda_{i, j, k, 7}$.

Table 3 - Example of inter layer emergent behaviour characteristics

\begin{tabular}{lllllllll}
\hline Signal & & $\lambda_{\boldsymbol{i}, \boldsymbol{j}, \boldsymbol{k}, \mathbf{1}}$ & $\boldsymbol{\lambda}_{\boldsymbol{i}, \boldsymbol{j}, \boldsymbol{k}, \mathbf{2}}$ & $\boldsymbol{\lambda}_{\boldsymbol{i}, \boldsymbol{j}, \boldsymbol{k}, \mathbf{3}}$ & $\boldsymbol{\lambda}_{\boldsymbol{i}, \boldsymbol{j}, \boldsymbol{k}, \mathbf{4}}$ & $\boldsymbol{\lambda}_{\boldsymbol{i}, \boldsymbol{j}, \boldsymbol{k}, \mathbf{5}}$ & $\boldsymbol{\lambda}_{\boldsymbol{i}, \boldsymbol{j}, \boldsymbol{k}, \mathbf{6}}$ & $\boldsymbol{\lambda}_{\boldsymbol{i}, \boldsymbol{j}, \boldsymbol{k}, \mathbf{7}}$ \\
\hline $\boldsymbol{\gamma}_{\mathbf{1 , 2 , \mathbf { 1 }}}$ & $\boldsymbol{F C}_{\text {inst }}$ & 0.89222 & 0.6818 & 0.94293 & 0.89017 & 0.94724 & 0.89037 & 0.98015 \\
$\boldsymbol{\gamma}_{\mathbf{1 , 3 , 2}}$ & $\boldsymbol{T}_{\text {eng }}$ & 0.90433 & 0.61222 & 0.91731 & 0.85698 & 0.93967 & 0.85703 & 1 \\
$\boldsymbol{\gamma}_{\mathbf{1 , 3 , \mathbf { 1 }}}$ & $\boldsymbol{N}_{\text {eng }}$ & 0.96077 & 0.61245 & 0.90673 & 0.85285 & 0.94612 & 0.85387 & 1 \\
\hline
\end{tabular}

The value of $\lambda_{\mathrm{i}, \mathrm{j}, \mathrm{k}, 7}$ drops moving up through the system levels through the model, indicating an escalation of the accumulated error. If at any point within the signal decomposition hierarchy the value for $\lambda_{\mathrm{i}, \mathrm{j}, \mathrm{k}, 7}$ is higher than the signal(s) at the level below, this means that there has been an 'averaging out' effect where two or more incorrect signals have combined to produce a higher level signal that has (by chance) a more 'correct' value in relation to the reference results.

\subsection{Global Validation Metric}

While each of the previous steps provides an important insight into the various quality measures of the system, it is proposed here that these measures can be combined to 
present a global validation metric, in which each of the previous metrics is nested. This metric will serve to summarise the validation characteristics of the model relative to the defined behavioural set and provide a global measure of the simulation quality. This proposed global validation metric is calculated as shown in equation 14.

$$
\Phi=\frac{1}{\sum_{i=1}^{I} \sum_{j=1}^{J} \sum_{k=1}^{K} \alpha_{i, j, k}} \sum_{i=1}^{I} \sum_{j=1}^{J} \sum_{k=1}^{K} \alpha_{i, j, k}\left(\frac{1}{\sum_{q=1}^{Q} \beta_{q}} \sum_{q=1}^{Q} \beta_{q} \lambda_{i, j, k, q}\right)
$$

where $I$ is the total number of signal sets, $J$ is the total number of decomposition levels within a respective set, $K$ is the total number of signals at a given level of decomposition within a set, $\alpha_{i, j, k}$ is the weighting factor applied to signal $\mathrm{i}, \mathrm{j}, \mathrm{k}, Q$ is the total number of behavioural characteristics, and $\beta_{q}$ is the weighting factor applied to the $q^{\text {th }}$ validation metric. Each signal is weighted relative to the importance of the individual signal to the validation, $\alpha$, and relative behavioural characteristic, $\beta$. It is at this point in the process that some subjectivity is inevitable as the user must decide how to weight each signal and metric. Again, an example of a fraction of the powertrain signals is shown in Table 4, with each of the behavioural characteristics calculated. 
Table 4 - Results from behavioural characteristic analysis

\begin{tabular}{|c|c|c|c|c|c|c|c|c|c|c|}
\hline$\overline{\text { Set }}$ & Signal & Name & $\alpha$ & $\lambda_{i, j, k, 1}$ & $\overline{\lambda_{i, j, k, 2}}$ & $\lambda_{i, j, k, 3}$ & $\lambda_{i, j, k, 4}$ & $\overline{\lambda_{i, j, k, 5}}$ & $\overline{\lambda_{i, j, k, 6}}$ & $\overline{\lambda_{i, j, k, 7}}$ \\
\hline $\bar{\beta}$ & & & - & 1 & 1 & 1 & 1 & 1 & 1 & 1 \\
\hline \multirow[t]{4}{*}{$\Gamma_{1}$} & $\gamma_{1,1,1}$ & $F C_{\text {cum }}$ & 1 & 0.99989 & 0.8615 & 0.98428 & 0.97936 & 0.99508 & 0.98662 & 0.92120 \\
\hline & $\gamma_{1,2,1}$ & $F C_{\text {inst }}$ & 0.7 & 0.88149 & 0.6818 & 0.94293 & 0.89017 & 0.94724 & 0.89037 & 0.97939 \\
\hline & $\gamma_{1,3,2}$ & $T_{e n g}$ & 0.5 & 0.88833 & 0.58423 & 0.91731 & 0.85698 & 0.93967 & 0.85703 & 1 \\
\hline & $\gamma_{1,3,1}$ & $N_{e n g}$ & 0.5 & 0.95837 & 0.59177 & 0.90673 & 0.85285 & 0.94612 & 0.85387 & 1 \\
\hline$\Gamma_{2}$ & $\gamma_{2,1,1}$ & $v$ & 1 & 0.99903 & 0.93321 & 0.98761 & 0.97807 & 0.99046 & 0.97949 & 1 \\
\hline
\end{tabular}

Global Validation Metric Value (Ф)

0.9275

If the global validation metric is calculated using the results in table 4 and equation 14, the overall validation metric for the simulation with given conditions, $\Phi$, is 0.9297 . This number is representative of each of the behavioural characteristics as each is nested within the global validation metric, weighted by what the user has interpreted to be of higher importance for the model's application. It was decided to use high precision on the individual metrics to ensure that even small differences in magnitude could be captured when calculating the global validation metric. If desired the user can then round this value post calculation. For example, in this case the global validation metric could be rounded to 0.93. 


\section{Discussion}

The design of the next generation hybrid vehicles presents challenges when attempting to understand many of the subtilties in the underlying energy flow patterns, as the volume of data available (often in the order of $10^{8}+$ data points) can often obscure whether the mathematical model generated accurately represents the real-life vehicle performance. As concerns about energy management and emissions have come to the forefront, these detailed validation procedures have become necessary in order to design robust control strategies which can maximise the energy consumption efficiency. While it is a (comparatively) trivial task to validate global vehicle characteristics, such as total fuel consumption, it is a monumental undertaking to compare and understand the accuracy with which the full range of vehicle behaviours are being accurately captured for these new generations of hybrid vehicles. Use of a typical validation approach in this instance would result in a set of error values as outlined in table 5. However, these metrics give no indication of the suitability of the model's instantaneous behavioural characteristics and only assess the simulation results on a global basis. 
Table 5 - Validation results using typical metrics

\begin{tabular}{cc}
\hline Metric & Result \\
\hline Error at end of simulation & $141.76 \mathrm{ml}(2.6 \%)$ \\
RMS & $42.52 \mathrm{ml}$ \\
Error Standard Deviation & $56.10 \mathrm{ml}$ \\
\hline
\end{tabular}

This paper has first introduced the core set of behaviours that any advanced vehicle powertrain should be evaluated against, which not only gives a measure of the magnitude of any error within the model, but aids in identifying the likely source of those errors to aid in improving underlying modelling strategies. For example, the cross correlation presented can identify time domain phasing errors, which would not naturally be obvious in standard error evaluations. These behavioural profiles for all of the signals in the vehicle architecture provides the necessary additional insight needed to understand what aspects of the model require modification and/or refinement post-validation. However, large tables of values can still present a challenge, and a more visual representation of this validation is likely to be more useful. In Figure 10, the various behaviours for the sample model in Table 4 are shown. For each signal in the system, the $\mathrm{x}$-axis gives the assigned 'validation value' for each signal in the system, the y-axis provides the weighting assigned to that signal/behaviour combination $(\alpha x \beta)$, and the relative radii of each glyph indicates 
the position within the hierarchy (largest - Level 1, smallest, Level 3). The glyph fill indicates the behaviour 'type' $\left(\lambda_{i, j, k, 1}\right.$ to $\left.\lambda_{i, j, k, 7}\right)$.

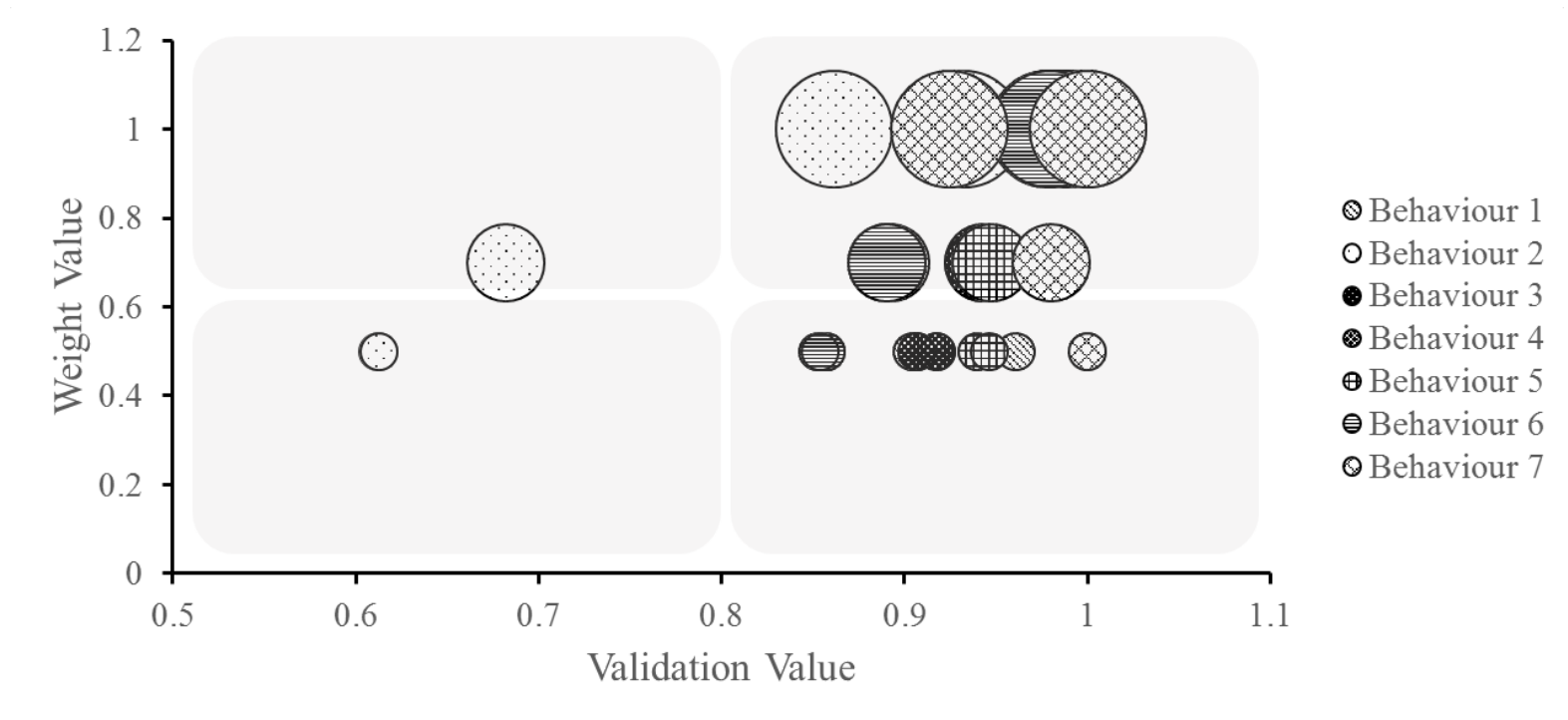

Figure 10 - Visual representation of behavioural results

The key aspect of this figure is the quick and easy identification of outliers (and hence the undesirable behaviours within the vehicle model), which may be obscured otherwise. In the case above, it is easy to see that across the model, there is a strong level of correlation with nearly all signal/behaviour combinations across the full extent of the system (evidenced by the clustering in the top righthand corner), but there are issues at lower levels with the first order derivative matching $\left(\lambda_{i, j, k, 2}\right)$ at the lower system levels which may require some additional investigation (bottom left). By presenting this information in an intuitive manner, along with all the appropriate data sets, it assists the user with making informed decisions about the suitability of the vehicle model for control system 
development/refinement. The validation of the mild-hybrid vehicle model using the proposed algorithm has played a crucial role in vehicle development. It has increased confidence in the model results, meaning that development work, especially on the energy management strategy, has progressed more rapidly. This has ultimately led to the vehicles produced, both single and double deck variants, being certified to low carbon emission bus status by the Low Carbon Vehicle Partnership (LowCVP) [32], [33].

\section{Conclusions}

A multi-fidelity validation algorithm has been developed and tested on a next generation mild-hybrid bus configuration. To support the development of highly efficient vehicle control strategies which can efficiently minimise energy consumption, it is first necessary to have a well validated model which can be easily refined with a high level of confidence, where all underlying energy flow paths and system dynamics are being suitably captured. To this end, seven critical signal behaviours have been identified which should be evaluated in the vehicle validation process, which are capable of fully representing not only the full range of likely errors associated with the vehicle model, but also guide the user towards the root cause. By presenting the behavioural characteristic plot, outliers can be quickly identified, assisting in the rapid identification of areas of poor correlation in the system and the likely underlying source. A global validation metric has been proposed and tested, which provides an abstract representation of the model validation quality, taking into consideration all relevant global and local characteristics, nested into a single 
value. By approaching the validation in this manner, the subjectivity which is typically inherent in many published validation approaches is delayed until as late as possible, removing the potential for unintentional bias. Through the combination of a global metric and visual representation of the full range of model behaviours baselined against the physical system, the quality of representation of the underlying system dynamics across all levels of the model is more transparent, and it is also possible to use these metrics and visualisations to more effectively understand the effects of post-validation changes to the system on the model quality. The validation process has played a significant role in vehicle development with the vehicles considered in the study achieving low carbon emission bus status from the LowCVP, an organisation that promotes a sustainable shift towards low carbon vehicles in the UK.

\section{Funding}

This work was supported by the Northern Ireland Department for the Economy (DfE) and the Wrights Group Ltd.

\section{Conflict of Interest}

The Authors declare that there is no conflict of interest

\section{References}

[1] O. F. Delgado, N. N. Clark, and G. J. Thompson, "Heavy Duty Truck Fuel Consumption Prediction Based on Driving Cycle Properties,” Int. J. Sustain. Transp., 2011. 
[2] I. Briggs, M. Murtagh, R. Kee, G. McCullough, and R. Douglas, "Sustainable nonautomotive vehicles: The simulation challenges," Renew. Sustain. Energy Rev., vol. 68, pp. 840-851, 2017.

[3] J. Rios-Torres, J. Liu, and A. Khattak, "Fuel consumption for various driving styles in conventional and hybrid electric vehicles: Integrating driving cycle predictions with fuel consumption optimization,” Int. J. Sustain. Transp., 2018.

[4] The European Parliament and the Council of the European Union, "REGULATION (EC) No 595/2009 OF THE EUROPEAN PARLIAMENT AND OF THE COUNCIL of 18 June 2009 on type-approval of motor vehicles and engines with respect to emissions from heavy duty vehicles (Euro VI) and on access to vehicle repair and maintenance informati," Off. J. Eur. Union, vol. 52, no. L 188, 2009.

[5] The European Comission, "COMMISSION REGULATION (EU) No 582/2011 of 25 May 2011 implementing and amending Regulation (EC) No 595/2009 of the European Parliament and of the Council with respect to emissions from heavy duty vehicles (Euro VI) and amending Annexes I and III to Direct," Off. J. Eur. Union, vol. L 167, 2011.

[6] O. Balci, "Verification, Validaiton and Accreditation of simulation models," Proc. 1997 Winter Simul. Conf., pp. 135-141, 1997.

[7] S. Groesser and M. Schwaninger, "Contributions to model validation: hierarchy, process, and cessation," Syst. Dyn. Rev., vol. 28, no. 2, pp. 157-181, 2012.

[8] R. G. Sargent, "Verification and Validation of Simulation Models," Winter Simul. Conf., pp. 124-137, 2007.

[9] W. L. Oberkampf and T. G. Trucano, Verification and validation in computational fluid dynamics, vol. 38, no. 3. 2002. 
[10] M. Martis, "Validation of simulation based models: A theoretical outlook," Electron. J. Bus. Res. Methods, vol. 4, no. 1, pp. 39-46, 2006.

[11] I. O. for Standardization, “ISO 19365:2016," 2016. [Online]. Available: https://www.iso.org/standard/64702.html. [Accessed: 04-Aug-2017].

[12] ASME, "Guide for verification and validation in computational solid mechanics," ASME Stand. V\&V 10-2006, pp. 1-15, 2006.

[13] R. G. Sargent, "Verification and validation of simulation models," Proc. 2011 Winter Simul. Conf., pp. 2194-2205, 2011.

[14] O. Topcu, "Review of Verification and Validation Defence R \& D Canada," 2003.

[15] U. Kiencke and L. Nielsen, Automotive Control Systems, For Engine, Driveline, and Vehicle, 2nd ed. Springer-Verlag, 2005.

[16] F. Ahmad, S. A. Mazlan, H. Zamzuri, H. Jamaluddin, K. Hudha, and M. Short, "Modelling and Validation of the Vehicle Longitudinal Model," Int. J. Automot. Mech. Eng., vol. 10, no. December, pp. 2042-2056, 2014.

[17] A. Brooker, J. Gonder, L. Wang, E. Wood, S. Lopp, and L. Ramroth, "FASTSim : A Model to Estimate Vehicle Efficiency, Cost and Performance," SAE Tech. Pap., no. April, pp. 21-23, 2015.

[18] H. Dourra and A. Mouratad, "Design and Control of Transmission Systems using Physical Model Simulation," SAE Tech. Pap., 2010.

[19] S. W. Hunt, a. M. C. Odhams, R. L. Roebuck, and D. Cebon, "Parameter measurement for heavy-vehicle fuel consumption modelling," Proc. Inst. Mech. Eng. Part D J. Automob. Eng., vol. 225, no. 5, pp. 567-589, 2011.

[20] S. Leach and M. Jennings, "Powersplit HEV Performance Simulation Capability," SAE Tech. Pap., 2014. 
[21] F. Millo, R. Fuso, L. Rolando, J. Zhao, A. Benedetto, F. Cappadona, and P. Seglie, "HYBUS: A new hybrid bus for Urban Public Transportation," SAE Tech. Pap., vol. $6,2013$.

[22] L. Damiani, M. Repetto, and A. P. Prato, "Improvement of powertrain efficiency through energy breakdown analysis," Appl. Energy, vol. 121, pp. 252-263, 2014.

[23] C. Fiori, K. Ahn, and H. A. Rakha, "Power-based electric vehicle energy consumption model: Model development and validation," Appl. Energy, vol. 168, pp. 257-268, 2016.

[24] M. R. Gaballo, M. Giodice, A. Diano, F. Fersini, F. Miccolis, S. Mannal, and S. Motz, “Application of a Modular Simulation Approach : Optimizations from Combustion to Vehicle Management," SAE Int., no. 2015-24-2505, 2015.

[25] A. Walker, A. McGordon, G. Hannis, A. Picarelli, J. Breddy, S. Carter, A. Vinsome, P. Jennings, M. Dempsey, and M. Willows, "A novel structure for comprehensive HEV powertrain modelling," 2006 IEEE Veh. Power Propuls. Conf. VPPC 2006, pp. 1-5, 2006.

[26] N. K. Pandey, "Novel Approach for Model-Based Development - Part II : Developing Virtual Environment and Its Application," SAE Tech. Pap., 2016.

[27] J. Wang, H. A. Rakha, and C. E. Via, "Fuel consumption model for conventional diesel buses," Appl. Energy, vol. 170, pp. 394-402, 2016.

[28] G. J. Heydinger, M. K. Salaani, W. R. Garrott, and P. A. Grygier, "Vehicle dynamics modelling for the National Advanced Driving Simulator," Proc. Inst. Mech. Eng. Part D, 2002.

[29] R. Wade-Allen, J. P. Chrstos, G. Howe, D. H. Klyde, and T. J. Rosenthal, "Validation of a non-linear vehicle dynamics simulation for limit handling," Proc. 
Inst. Mech. Eng. Part D, 2002.

[30] J. Klemmer, J. Lauer, F. Volker, R. Fontaine, P. Kilian, S. Sinsel, A. Erbes, and J. Zapf, "Definition and Application of a Standard Verification and Validation Process for Dynamic Vehicle Simulation Models," SAE Int. J. Mater. Manuf., vol. 4, no. 1, pp. 743-758, 2011.

[31] G. Stevens, M. Murtagh, R. Kee, J. Early, R. Douglas, and R. Best, "Development of a Vehicle Model Architecture to Improve Modeling Flexibility,” SAE Int. J. Engines, vol. 10, no. 3, pp. 2017-01-1138, 2017.

[32] LowCVP, "Low emission bus scheme Certificate-Wrightbus Streetlite - H.E.V.," 2017. [Online]. Available: https://www.lowcvp.org.uk/Hubs/leb/LEBCertificates.htm\#Wrightbus.

[33] LowCVP, "Low emission bus scheme Certificate-Wrightbus Streetdeck - H.E.V.," 2017. [Online]. Available:

https://www.lowcvp.org.uk/Hubs/leb/LEBCertificates.htm\#Wrightbus. 